\title{
Explorations to improve the completeness of exome sequencing
}

\author{
Chen Du, Barbara N. Pusey, Christopher J. Adams, C. Christopher Lau, William P. Bone, William A. Gahl, \\ Thomas C. Markello and David R. Adams*
}

\begin{abstract}
Background: Exome sequencing has advanced to clinical practice and proven useful for obtaining molecular diagnoses in rare diseases. In approximately $75 \%$ of cases, however, a clinical exome study does not produce a definitive molecular diagnosis. These residual cases comprise a new diagnostic challenge for the genetics community. The Undiagnosed Diseases Program of the National Institutes of Health routinely utilizes exome sequencing for refractory clinical cases. Our preliminary data suggest that disease-causing variants may be missed by current standard-of-care clinical exome analysis. Such false negatives reflect limitations in experimental design, technical performance, and data analysis.

Results: We present examples from our datasets to quantify the analytical performance associated with current practices, and explore strategies to improve the completeness of data analysis. In particular, we focus on patient ascertainment, exome capture, inclusion of intronic variants, and evaluation of medium-sized structural variants.

Conclusions: The strategies we present may recover previously-missed, disease causing variants in second-pass exome analysis. Understanding the limitations of the current clinical exome search space provides a rational basis to improve methods for disease variant detection using genome-scale sequencing techniques.
\end{abstract}

Keywords: Clinical exome sequencing, Analytical quality, Performance enhancement, Clinical genomics, Rare diseases, Completeness problem, False negative results

Abbreviations: UDP, Undiagnosed diseases program; NIH, National Institutes of Health; NHGRI, National Human Genome Research Institute; HPO, Human phenotype ontology terms; NISC, NIH intramural sequencing center; CCDS, Consensus coding sequence project; UCSC, University of California Santa Cruz; MAF, Minor allele frequency; CADD, Combined annotation dependent depletion; HGMD, Human gene mutation database; SNV, Single nucleotide variant; CNV, Copy number variant; SNP, Single nucleotide polymorphism

\section{Background}

The Undiagnosed Diseases Program (UDP) of the National Institutes of Health (NIH) was established in 2008 to evaluate patients who were undiagnosed despite an extensive medical workup [1-3]. Besides thorough clinical phenotyping by multiple specialists, the UDP has been utilizing exome sequencing and SNP array analysis when a genetic etiology is suspected. The feasibility of using exome sequencing to identify new disease genes was first demonstrated in $2009[4,5]$ and has ever since contributed to the discovery of many Mendelian disease genes

\footnotetext{
* Correspondence: dadams1@mail.nih.gov

$\mathrm{NIH}$ Undiagnosed Diseases Program, Common Fund, National Institutes of Health, National Human Genome Research Institute, Bethesda, MD, USA
}

[6]. Exome sequencing studies have become an increasingly routine clinical approach with a reported $25 \%$ molecular diagnostic rate $[7,8]$. In the past few years, we observed that an increasing percentage of pediatric patients referred to the UDP has already been studied with a clinical exome. Hence, we employed an extended set of analytic approaches to identify disease-causing variants beyond those detected by current clinical exome analysis.

Several specific features of an exome analysis pipeline contribute to the final sensitivity and specificity of the overall test. They include the design of the analysis, e.g., included family members, limitations of the underlying sequencing technology, and a number of specific analytic 
parameters that affect variant filtration and prioritization. These include segregation rules, application of allele frequency cutoffs derived from control populations, and predictions of deleteriousness $[9,10]$. The choice of analytic parameters depends on the experimental design and scope of testing, with an overall goal of optimizing the final list of prioritized variants to be subjected to manual curation. Stringent parameters, focusing only on coding sequences in known disease genes and common Mendelian inheritance models, may filter out a "true" variant and create false negative results. This approach is commonly applied in clinical exome analysis where clinical interpretability is prioritized over new gene discovery. Conversely, relaxed filtration settings feature enhanced sensitivity but generate a number of false positive variants that increase the work associated with final curation. This approach is generally more suitable to a research-level analysis, where variants in genes not yet associated with disease may be of intrinsic interest.

Our current goal is to optimize research-level exome analysis for single, small-pedigree families. We quantify the consequences of widening the final search space using several analytic techniques. They include sequencing additional family members; evaluation of minimal exome coverage; reducing the number of false negative results by considering variants in non-coding regions; and searching for medium-sized indels missed by the standard genotyping modules of current analytical pipelines. Each of these potential second-pass procedures can be employed when standard exome analyses fail to provide a satisfactory explanation for the patient's clinical features.

\section{Results}

\section{Patients}

A growing number of pediatric patients referred to the UDP present with prior unrevealing clinical exome studies (Fig. 1a), which necessitates the development of novel diagnostic strategies to improve experimental power. Cohort-based studies, which have been successful in detecting disease genes by exome sequencing $[4,5]$, are not an appropriate tool to employ for our subjects given their high degree of phenotypic diversity as demonstrated by comparison of Human Phenotype Ontology terms $[11,12]$ that were used to characterize each affected patient (Fig. 1b). Additionally, most of our cases are limited to a nuclear family that is too small to achieve the LOD score thresholds used in linkage analyses (Fig. 1c). These factors have focused our attention on the general problem of maximizing the information that can be extracted from small-family, $n=1$ cases [13].

\section{Ascertainment of family members}

Our study participants have undergone extensive medical testing before being accepted into our program. As a result, many easily-identifiable diagnoses, and interpretable DNA sequence variants, have been previously excluded in patients of the UDP cohort, in particular when patients had undergone prior clinical exome testing. Therefore, our patients require an exome sequencing strategy optimized for agnostic testing, going beyond a clinical routine analysis pipeline, which is optimized for interpretable results and identifying variants in known disease genes.

A typical exome analysis pipeline includes an unsupervised variant filtration component. This may be followed by manual BAM file inspection, manual bioinformatic curation and expert clinical evaluation. Practice varies with regard to the number of family members included in exome sequencing and analysis. The value of family trios is increasingly recognized $[8,11]$; sequencing of all available siblings has been a common practice in the UDP [14].

To study the effect of included family members on exome analysis, we performed a standard variant filtration analysis on 45 families, while varying the family composition. These families included the proband, both unaffected parents and at least two additional siblings (at least a quintet in total). Taking different numbers of family members into account (Fig. 2), we

Fig. 1 Characteristics of the UDP patient cohort. a Percentage of pediatric cases ( $n=11$-28 per quarter year) with prior inconclusive exome
sequencing that applied to the UDP. b Number of HPO terms that are shared by a given number of patients (total $n=350$ affected individuals).
The top five HPO terms that were used in more than 50 patients were spasticity, global developmental delay, gastroesophageal reflux, seizures
and short stature. c Family structures of nuclear families in the UDP cohort, $n=329$




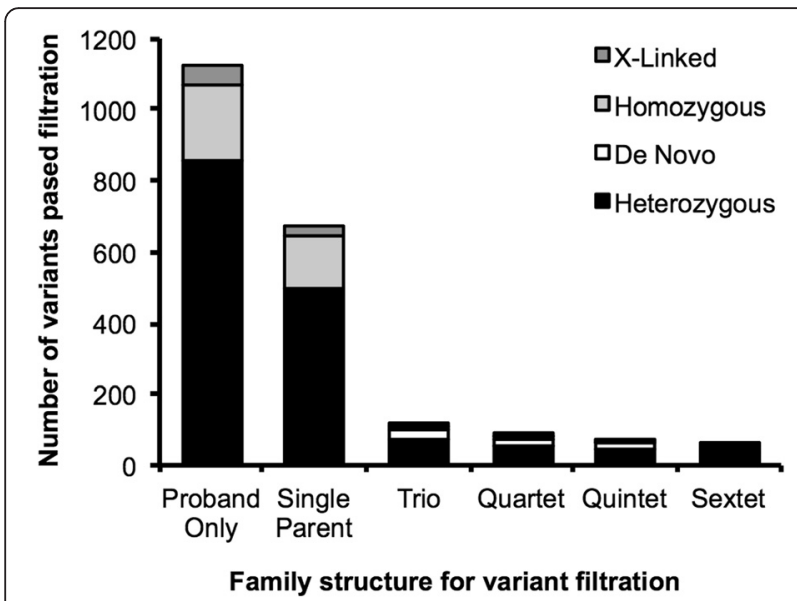

Fig. 2 Effects of family members on the number of variants returned by computerized filtration. Variants of 36 quintets and 9 sextet families were analyzed with an increasing number of family members. Bars show average number of variants that passed a basic filtration algorithm for segregation with disease, population frequency and transcript effects, based upon various inheritance models

filtered variants for segregation with disease, population frequency and transcript effects, but did not filter for known disease genes, in silico predictions of deleteriousness, or family-based linkage. The difference in the number of variants returned for manual evaluation was most striking between a singleton and a trio analysis, with about 1,126 (range 886-1521) vs. 117 (range 59-265) variants passing automated filtration, similar to the 10 -fold reduction reported previously [11]. A large part of this effect is attributable to a decrease in the number of heterozygous variants. In a trio, such variants could be assessed for de novo occurrence in light of parental genotypes and/or phased correctly as compound heterozygous variant pairs. The number of false positive homozygous recessive variants was greatly reduced by the awareness of parental genotypes. Additional number of siblings beyond a trio provided a further reduction in the average number of variants down to 88 (range 22-209), 69 (range 8-171) and 54 (range 11-109) for quartets, quintets and sextets, respectively. These reductions were obtained even without mapping recombination sites [15], by which siblings can exclude genomic regions from consideration and therefore remove large numbers of false positive variants.

In our research setting, the increased filtration power of additional informative meioses in family members balanced against the effort required to obtain the correct affected status, and the financial cost of sequencing and data processing. We justified this approach as being worthwhile in order to reduce the number of false positive variants when analyzing individual families $(n=1)$, so that time and effort could instead be used to reduce the extent of false negative results and therefore to improve completeness of analysis.

\section{Exome coverage}

Capture and enrichment of the exome for medical sequencing limits most of the analysis to the protein coding sequences -about 1-2 \% of all 3.2 billion base positions in the human genome- where the majority of previously documented disease-causing variants occur [16]. To assess loss of data due to lack in exome capture, we selected a subset of exomes that included 54 probands of the UDP cohort. Each member of this subset was sequenced under the same conditions at one sequencing center, and aligned through the same pipeline.

When coverage is based on the targeted regions as determined by the capture kit (TruSeq, about $61 \mathrm{Mb}$ ), we observed a mean coverage of 76 reads and a median coverage of 57 reads. $85 \%$ of targeted positions were covered more than $20 x$, a coverage frequently used as a minimal read depth requirement for confident genotyping of both alleles. Since capture kits differ in their target regions, we evaluated the coverage of all exonic regions defined by CCDS (about $31 \mathrm{Mb}$ ), as a measure of desired capture. Here we observed a mean coverage of 82 reads (range 44-130) and a median coverage of 61 reads (range 32-110), with an average of $88 \%$ (range 74-94) of exonic positions covered more than $20 \times$ (Fig. 3a, b).

Next we examined coverage based on CCDS exons instead of individual positions and grouped all exons into categories based on the minimum number of reads occurring in a given exon. This analysis showed that an average of $2.2 \%$ of exons (range 1.6-2.8) had no coverage at all, $2.1 \%$ (range 1.6-2.8) of exons had partially no coverage, $6 \%$ (range 2-17) showed a minimum coverage of fewer than 10 reads, and $12 \%$ (range 2-32) fewer than 20 reads (Fig. 3c). In total, an average of $10 \%$ of exons (range 6-21) had a minimum coverage of fewer than 10 reads and $22 \%$ (range 8-53) of exons had a read depth of fewer than 20 reads in at least one position (Fig. 3d). Of these low coverage exons, an average of more than $25 \%$ (range 24-30) were in genes known to harbor disease-causing or likely disease-causing variants (HGMD classes "DM" and "DM?"). In addition, coverage below 10 reads and below 20 reads was observed in $27 \%$ (range 19-38) and $42 \%$ (range 26-63) of first exons, respectively (Fig. 3e), indicating a notable contribution of first exons to the low coverage regions of exon sequencing, as generally recognized. When analyzed by entire genes (total number $n=18.351$ for females, $n=$ 18.409 for males), an average of $56 \%$ (range 39-78) and $76 \%$ (range 51-94) of genes had a minimum coverage below a depth of 10 and 20 reads, respectively (Fig. 3f), suggesting that low coverage occurred across the entire length of genes.

To estimate the impact of insufficient coverage on the diagnosis of known diseases, we queried the read depth at positions of known disease-causing variants listed in 


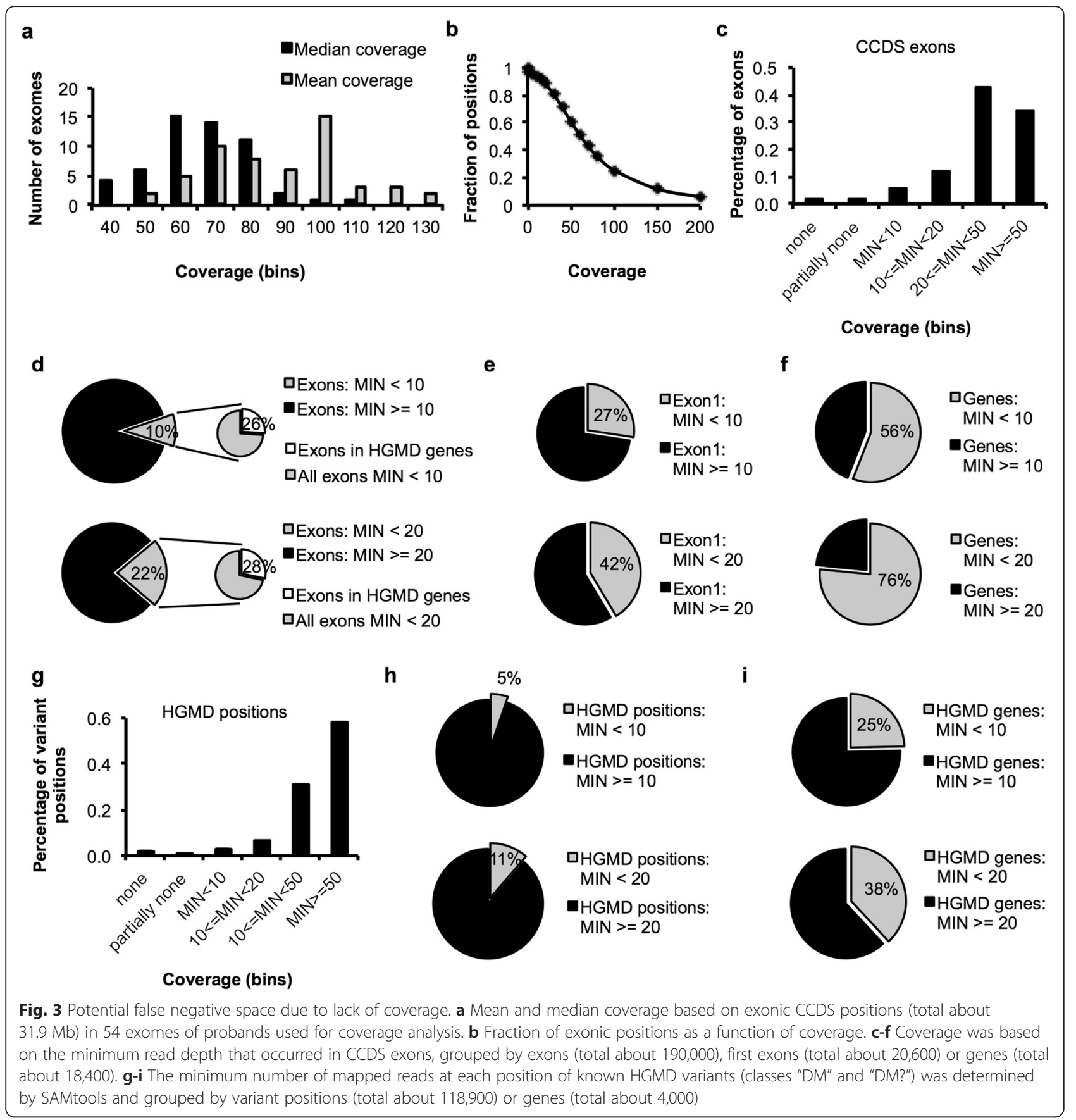

HGMD ( $n=118.861$ positions for females, $n=118.949$ positions for males). This subset of positions showed a higher coverage with only $5 \%$ (range $3-11$ ) of known disease-causing variant positions covered less than $10 \times$, and $11 \%$ (range 4-31) were covered with fewer than 20 reads (Fig. 3g-i). Low coverage variant positions of a read depth of less than $10 \times$ occurred in about $25 \%$ (range 17-38) of all HGMD genes and in $38 \%$ (range 22-62) based on a coverage of less than $20 \times$.
In a next step we analyzed the consistency of coverage across proband samples. Of 64,818 autosomal exons that showed read depths below 10 reads, 8199 exons were affected by low coverage in all 54 probands, of which 4406 exons were within the target sequence of the capture kit used (list of exons in Additional file 1: Table S1). When analyzed by genes, 1304 genes were covered with fewer than 10 reads in all 54 probands in more than $25 \%$ of their exons. In these genes, all low coverage exons in 
841 genes were within the targeted region of the capture kit used (list of genes in Additional file 1: Table S2). Coverage below 10 reads in all exons occurred in 409 genes, of which 277 genes consisted of a single exon only.

While it is possible to characterize the number of variants in known disease genes that are missed due to incomplete capture, we hypothesize that variants in genes not yet associated with disease may be missed due to lack of coverage of one of the two alleles, especially if strict requirements for read depth are applied during data analysis.

\section{Non-exonic variants}

The majority of reported disease-causing variants reside in coding regions or canonical splice sites at exon/intron boundaries [16] but there are instances where deep intronic variants have been associated with disease [17-19] and genome sequencing has revealed evidence of selective pressure on intergenic and intronic regions, suggesting functional conservation [20]. Targeted re-analysis of candidate loci for non-coding variants has been successfully attempted [21]. However, non-coding variants are also detected as a result of off-target capture, and may be of high quality [22]. Therefore we quantified the analytical potential of targeted and off-target non-exonic variants, while exploring strategies to optimize the less-favorable signal to noise characteristics.

We analyzed variants called in 54 probands and their parents in non-exonic locations, defined as outside the UCSC exon regions (Fig. 4). On average, about 156,000 non-exonic variants were called in each proband (range from about 121,000 to 252,000 ). The median distance to the nearest exon was 169 bases (range 142-298) and the mean was 15,000 bases (range 11,600-25,700), with a maximal distance observed of 1.7 million bases on average. About $70 \%$ of variants were within a distance of 500 bases from the nearest exon boundary (Fig. 4a). The number of non-exonic variants dropped consistently at a distance of about 300-500 bases from the nearest exon (2.47 to 2.69 on the logarithmic scale in Fig. 4b). Given an average DNA libraries size of $280 \mathrm{bp}$, this cutoff appears to correspond to the maximal distance between a non-coding variant and targeted exonic sequence that occurred on either end of the same DNA fragment. This suggests that most non-exonic variants were sequenced as flanking regions of intended capture, rather than captured due to sequence similarity or presented as artifacts due to misalignment.

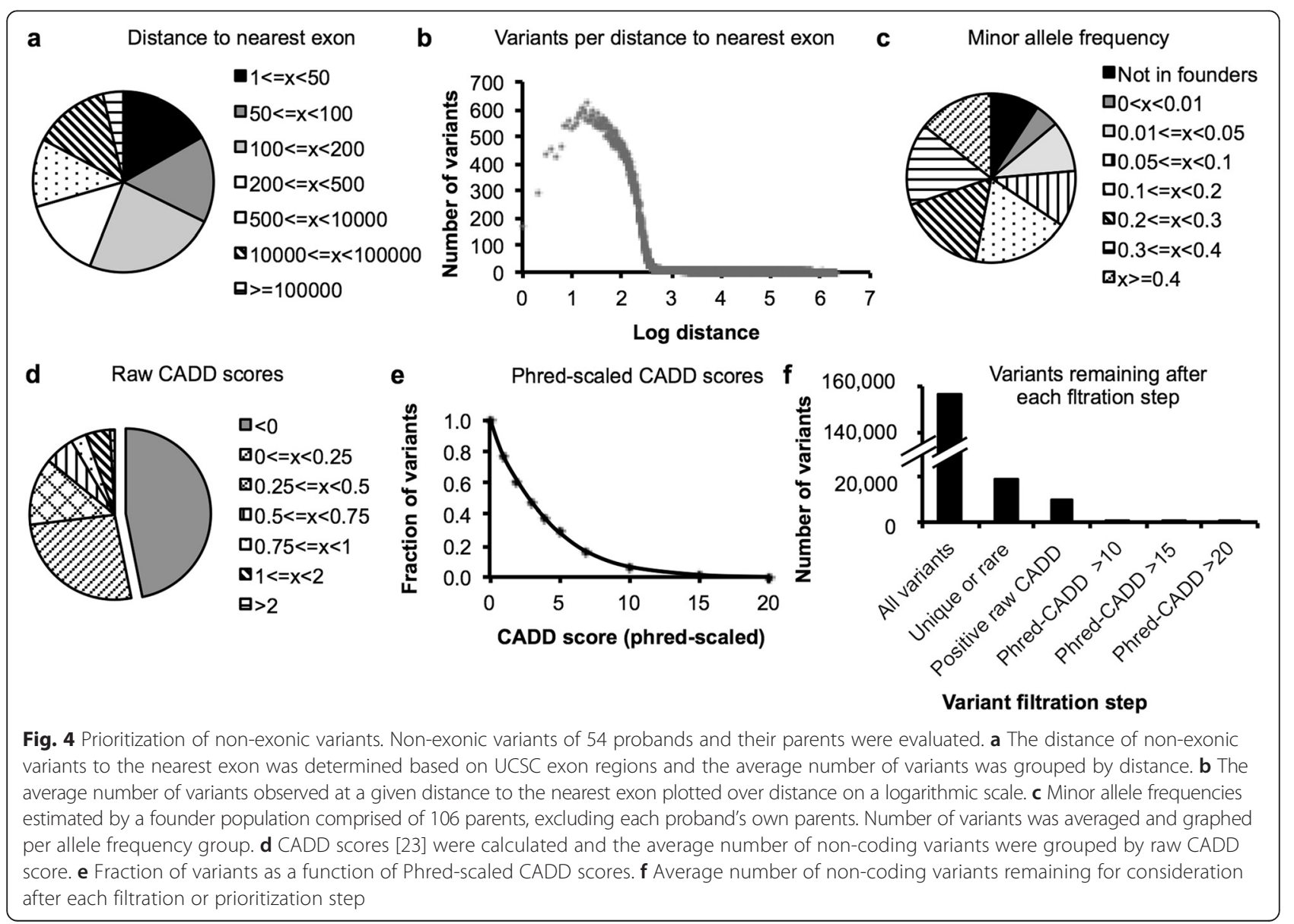


To filter the large number of non-coding variants, we estimated the minor allele frequencies based on a founder population comprised of 106 parents. We found that only $6 \%$ of the variants seen in each proband were absent from the founder population, which excluded each proband's own parents (Fig. 4c). Of variants present in the founder population, only $6 \%$ were rare at a $1 \%$ lower confidence interval limit of the estimated minor allele frequency. Overall, variant filtration based on allele frequency reduced the number of unique and rare nonexonic variants to 19,000 per proband on average (range from 7,500 - 89,000).

Prediction of deleteriousness is particularly difficult for non-coding variants since most prediction tools are limited to non-synonymous codon changes or canonical splice site positions. We tested the use of CADD scores [23] on our non-coding variant set, since this approach allows scoring of all SNVs and CNVs, not limited to coding variants. Raw CADD scores below zero were returned for $47 \%$ of all non-coding variants, indicating that these variants were not different from known benign variation (Fig. 4d). When we analyzed Phred-scaled CADD scores, we observed a mean score of 3.82, median of 2.84, a minimum score of 0.001 and a maximum of 36.5 on average. Only $7 \%$ of variants obtained a score higher than 10 and only $1 \%$ higher than 15 (Fig. 4e), indicating that a very low number of non-coding variants were actually predicted deleterious by CADD scores. Therefore, CADD scores could be an approach to highlight potentially interesting variants within the vast pool of mostly benign, but poorly annotated non-coding variants. More focused tools to predict splice site changes using multiple different algorithms and that can be incorporated into an automated computational pipeline should be able to prioritize additional variants for consideration.

Taking all information together, filtration based on allele frequency reduced the number of variants from initially 156,000 non-exonic variants per proband to 19,000 (Fig. 4f). Using a conservative approach to predict benign variants by negative raw CADD scores reduced the number of remaining variants to 9,400. Applying prediction of deleteriousness, an average of 1,200 variants were predicted damaging at a Phred-scaled CADD score of 10 . When more stringent filters were applied, only 285 variants on average obtained a CADD score higher than 15 and only 40 variants on average scored higher than 20. Depending on the desired stringency, we found that these filtration strategies generated a tractable number of additions to a routine second pass analysis.

\section{Medium-sized indel calling}

The limitations of genome-scale data analysis to identify structural variants is another known cause of false negative results. Standard variant callers typically identify indels up to about 50 bases. Supplementing exome diagnostics with SNP chip or array-CGH data is known to detect indels larger than a few kilobases genome wide. Therefore, a large range of medium-sized indels from 50 bases to a few kilobases remain unaccounted for in subsequent variant evaluation pipelines, contributing to incomplete analyses.

Many attempts have been made to address this issue by calling indels from exome sequencing data with additional methods [24-28], and such efforts have recently been implemented in large scale exome research studies [11]. While some programs examine read depth against a reference population, Pindel [29] extracts unmapped reads from BAM files and analyzes soft clipped bases of read pairs for evidence of medium-sized structural variation. We used Pindel to quantify the extent of incomplete analysis resulting from missed indels in a cohort of 54 probands and their parents.

Pindel detected on the order of 33,000 structural variants per proband on average (range about 22,000 to 54,000 ), of up to $+/-16,000$ bases in size (Fig. 5a). Half of these indels occurred within the target region defined by the capture kit and 50 bases of flanking regions (Fig. 5b). On average, $63 \%$ of all indels and $61 \%$ of indels within target regions were also detected by the variant caller and were therefore redundant (Fig. 5d). A breakdown by size showed that deletions of fewer than 50 bases and insertions of fewer than 10 bases were identified by both Pindel and the genotype caller in $56 \%$ to $76 \%$ of variants (Fig. 5 c). The overlap of called variants dropped rapidly beyond deletions larger than 100 bases and insertions larger than 50 bases.

We also used Pindel to call indels in the probands' parents and used their variants to estimate allele frequencies. This analysis revealed that only $5 \%$ of indels in the probands were absent from the control population (Fig. 5e). Of the indels that were present in the founder population (excluding each proband's own parents), $8 \%$ were rare at a $1 \%$ lower confidence interval limit of the estimated minor allele frequency. Thus, filtering all Pindel variants by allele frequency for unique or rare variants reduced the number of variants to about 4,200 on average (range from 1,500 - 11,700).

When we analyzed phasing of all Pindel variants of the proband, about $34 \%$ of all variants appeared to be inherited by one of the parents, $46 \%$ occurred in both parents and $20 \%$ could not be associated with an inheritance pattern (Fig. 5f). As expected, in a subset of variants that are only detected by Pindel and that are rare in or absent from the founder population, the percentage of variants that are present in both parents is greatly reduced (12\%), and enriched for variants not detected in the founder population (68\%). 


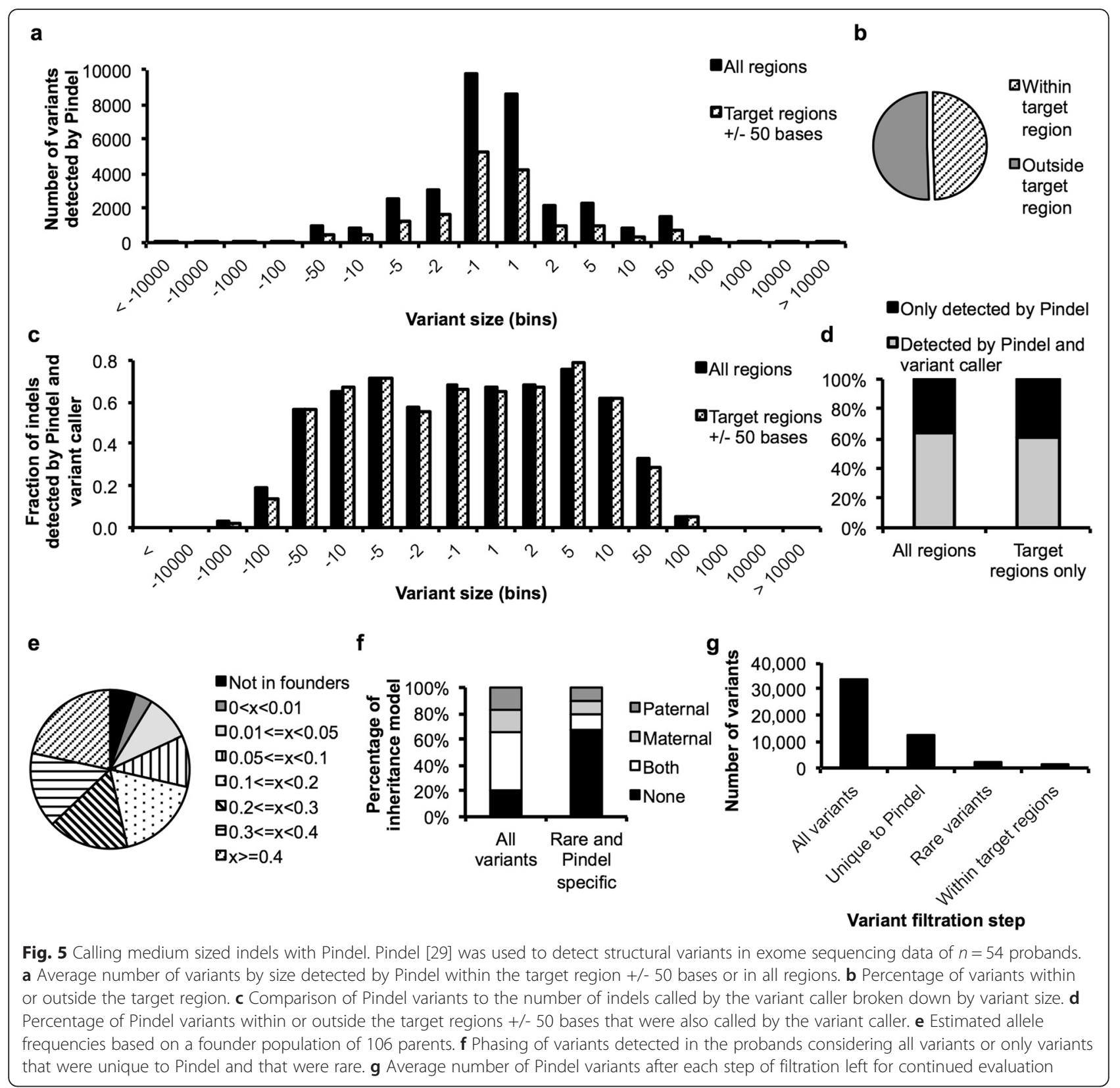

Combining all information gained on the indels called by Pindel (Fig. 5g), we started with an average of 33,000 Pindel variants per proband. An average of 12,200 variants were detected by Pindel only and not by the standard variant caller, of which an average of 2,600 variants were rare or unique indels in the proband by filtration based on founder frequencies (of which 1,200 variants map within target regions). This appears to be the lower limit that purely frequency-based bioinformatics tools can reach based on this cohort size, before variants are evaluated for other parameters such as Mendelian inheritance models and prediction of deleteriousness. With increased numbers and matched ethnicity of individuals in the control population, the power of filtration of ancient and benign variants based on allele frequency will improve and optimize computerized prioritization of indels when included in a second-pass analysis pipeline.

\section{Discussion}

In most clinical situations when exome sequencing is ordered as a diagnostic test, it is necessary and sufficient to limit the search parameters to well-defined areas of known disease genes and predictable protein changes. However, if such an attempt fails to reveal a molecular diagnosis and an agnostic research effort is made to discover potential new disease genes, non-Mendelian inheritance patterns, 
multi-gene conditions or unusual pathogenicity, it is desirable to broaden the analytical range to more obscure territories.

Widening search parameters to improve completeness of analysis by including non-exonic variants and medium sized indels may include the "true" variant that may be missed otherwise, but at the expense of increased noise. To handle the additional load of variants, we used the genotype data of 106 unaffected parents as a control cohort, under the assumption that the probands do not share the same rare condition. Although the power of filtration against large control populations, such as 1000 Genomes [30, 31], ESP (NHLBI GO Exome Sequencing Project, URL: http://evs.gs.washington.edu/ EVS/) or the ExAC database (Exome Aggregation Consortium, URL: http://exac.broadinstitute.org), is well recognized, we were impressed by the power of filtration that a relatively small cohort of 106 individuals sequenced under the same technical conditions, could provide for analyzing non-exonic variants and mediumsized indels. The use of genome sequencing information released with phase 3 of the 1000 Genomes project will further improve our ability to interpret the biological significance of SNVs and CNVs in non-exonic regions, such as in introns, promoter regions, UTRs, enhancers, intergenic regions and in transcribed non-coding DNA. This is especially important when the search parameters exceed coding regions and canonical splice sites, since most tools to predict deleteriousness are typically limited to these territories.

We characterized the lack of coverage in our exome sequencing studies, which is a generally recognized contributor of false negative results. The application of genome sequencing is known to provide a more homogenous coverage and therefore represents a true advance in the attempt to analyze all genes [32, 33]. However, even this technology lacks coverage at difficult to sequence, possibly non-mappable genomic regions [34]. Other technical advances, such as low amplification technologies (so called "PCR-free chemistry"), which minimizes allele dropout, is only available with larger DNA sample acquisition and whole genome sequencing chemistry.

In current exome sequencing, even highly medically important sets of genes, such as the 56 genes recommended by the American College of Medical Genetics and Genomics for clinical testing, can lack full coverage $[35,36]$. Spiking in extra baits enhances the capture of known disease genes. Although coverage has improved with newer capture kits [32], this approach appears inefficient when trying to fully capture all known genes [37], including those not yet associated with disease.

In addition to improved coverage, genome sequencing has also proven useful in the detection of small and large structural variants $[38,39]$, which may be missed by the standard variant calling pipelines used in exome sequencing. Advances in long read sequencing are hoped to provide optimized identification of structural variants and also allow detection of disease-causing tandem repeats [40-42].

In the future, it will be desirable to include other approaches to variant discovery into an analysis pipeline, beyond classical models of SNVs and CNVs that alter protein function. Examples include changes in DNA methylation [43, 44], disease-causing mobile elements [45] and the spatial organization of the genome [46-48], for example the disruption of long-range enhancer function or perturbation of insulators of topologically associating domains.

Once a research based exome sequencing experiment returns promising variants or novel disease gene candidates, demonstration of causality remains a challenge [10], especially given the typical $n=1$ family situation. Apart from functional studies to elucidate molecular mechanisms, identification of other similarly affected families will be key to validate research findings. Given the limited number of patients seen at a single clinical site, sharing data of both exome sequencing as well as clinical features, especially in standardized form using HPO terms that enable computerized phenotype matching, will become increasingly important [49]. Additionally, comparison of standardized phenotype information to model organisms and integration of pathway analyses have the potential to identify new disease genes for research studies [50-52].

\section{Conclusions}

We explored strategies to improve completeness of analysis in a research setting and demonstrated that the number of variants added is tractable when the search parameters are expanded to include non-coding variants and medium-size indels. Future applications of these approaches will establish to what degree these additional efforts contribute to the number of solved cases, in light of feasibility and efficiency, relative to the number of cases with inconclusive clinical exomes referred for research studies.

\section{Methods \\ Patients}

Patients of the UDP were enrolled in clinical protocol 76-HG-0238, "Diagnosis and Treatment of Patients with Inborn Errors of Metabolism and Other Genetic Disorders", approved by the Institutional Review Board of the National Human Genome Research Institute (NHGRI), and gave written informed consent. Human Phenotype Ontology terms (HPO) were used for standardization as part of clinical phenotyping $[12,53]$. The medical 
records of pediatric UDP applicants reviewed between 2013 and 2015 were screened to determine if exome or genome sequencing had been performed prior to evaluation by the UDP. To estimate family structures, a manually curated pedigree file containing all UDP participants who underwent molecular testing by SNP chip and/or exome sequencing was analyzed.

\section{Exome sequencing and analysis}

DNA was extracted from whole blood using the FlexStar system (Autogen). Libraries of $\sim 280 \mathrm{bp}$ and paired-end index adapters were prepared according to Illumina's TruSeq V1 or V2 method and sequenced at the NIH Intramural Sequencing center (NISC) on a HiSeq2000 sequencer (Illumina) using 101-bp paired-end reads. Alternatively, 275-325 bp DNA libraries were constructed using KAPA library preparation kit (KAPA), captured with SeqCap EZ Exome plus UTR Lib capture kit (Nimblegen) and sequenced at NISC on a HiSeq2500 (Illumina) using 126-bp paired-end reads. Other exomes were captured using TruSeq kit (Illumina) and sequenced at Axeq (Rockville, MD). For all exome sequencing experiments, short reads were aligned to human reference genome GRCh37 using an in-house developed pipeline based on Novoalign (Novocraft Technologies). Variants were called with HaplotypeCaller and GenotypeGVCFs [54-56]. Annotations utilized snpEff [57] and a combination of internal cohort statistics and publically available data sources (NHLBI GO Exome Sequencing Project (ESP), URL: http://evs.gs.washington.edu/EVS/, 1000Genomes [30]). Basic, computerized variant filtration was used to analyze the effects of family members on the number of variants returned. In brief, rare, nonsynonymous, start-gain/loss, stop-gain/loss, frameshift, canonical splice site variants and intronic variants (up to 20 bases from splice sites) were evaluated under homozygous recessive, compound heterozygous, $\mathrm{X}$-linked and de novo dominant disease models in families of European descent. A cohort of 54 cases, comprising of the proband and both parents, that were sequenced under the same conditions at NISC using TruSeqV2 capture was used for analysis of low coverage regions, noncoding variants, and medium-sized indels.

\section{Coverage analysis}

Positions within target regions, exons as annotated by the Consensus Coding Sequence project (CCDS) [58-60] or variant positions annotated as diseasecausing in the Human Gene Mutation Database (release 2014-1, classes "DM" and "DM?") [16] were queried for read depth using SAMtools in 54 probands of the UDP $[61,62]$. For per base coverage analysis, read depth at each unique position was considered. For exon- and gene-based analysis, the minimum read depth that occurred in a given exon was determined and used to group into categories.

\section{Non-exonic variants}

Non-coding variants outside the regions defined by UCSC exons (annotated in hg19 assembly by the University of California Santa Cruz [63]) were analyzed, while left-normalizing multiallelic variants. The parents of the 54 probands served as a control population, excluding parents of the proband who was examined $(n=106$ individuals, 212 alleles for autosomal variants, 159 alleles for $\mathrm{X}$-chromosomal variants, and 54 alleles for Ychromosomal variants). Absence of an alternative allele in the founders was treated as presence of a reference allele. To hypothesize that a variant was too frequently present in the control population to be causative for a rare disease, a cutoff of 6 or more variant alleles was used for autosomal alleles, which corresponds to a minor allele frequency (MAF) of 0.0283 (95\% confidence interval (CI) of 0.013 to 0.0604 using population proportion interval estimation [64]). For X-chromosomal variants, a cutoff at 5 or more alleles was used (MAF of 0.0316, $95 \%$ CI of 0.0136 to 0.0719$)$ and 2 or more variant alleles for Ychromosomal variants (MAF of $0.037,95 \%$ CI of 0.0102 to 0.1253 ). Variants in the probands were annotated with raw and Phred-scaled CADD v1.3 scores [23]. A negative raw score was used to assume that a variant was benign. Phred-scaled CADD scores were used to predict deleteriousness.

\section{Medium-sized structural variants in exome sequencing} Pindel [29] was used to detect medium-sized structural variants compared to GRCh37 assembly in 54 probands and their parents. The presence of supporting reads was interpreted as a heterozygous variant allele. Structural variants were compared to those called during pipeline genotyping, applying left-normalization of multiallelic variants. Parental data were used to determine the phase of variants in the probands. The parents of 54 probands served as a control population, excluding the parents of the proband who was examined. Under the assumption that all indels identified by Pindel are in heterozygous state, we applied the thresholds as stated above.

\section{Additional file}

Additional file 1: Table S1. Coverage of exons in 54 probands. Table S2. Genes with low coverage exons in all 54 probands. (XLSX 9613 kb)

Acknowledgements

We thank our patients and their families for their participation in the UDP, and we appreciate the contributions of our dedicated clinical team members. Special thanks to the bioinformatics team. 


\section{Funding}

This research is supported by the Intramural Research Program of the National Human Genome Research Institute and the Common Fund of the National Institutes of Health.

\section{Availability of data and materials}

All genomic data is in the process of being submitted to dbGaP. Once the submission is complete, the authors would be happy to supply a specific $\mathrm{dbGaP}$ Project ID on request.

\section{Authors' contributions}

$C D, B N P, C C L, T C M$ and DRM designed the study. CD, BNP, CJA and WPB wrote code for data analysis. CD, BNP and DRA interpreted data. CD, WAG, TCM and DRA wrote the manuscript. All authors reviewed and approved the final manuscript.

\section{Competing interests}

The authors declare that they have no competing interest.

\section{Consent for publication}

Not applicable since this manuscript does not contain any individual person's data.

\section{Ethics approval and consent to participate}

Patients of the UDP were enrolled in clinical protocol 76-HG-0238, "Diagnosis and Treatment of Patients with Inborn Errors of Metabolism and Other Genetic Disorders", approved by the Institutional Review Board of the National Human Genome Research Institute (NHGRI), and gave written informed consent.

Received: 30 November 2015 Accepted: 5 August 2016

\section{Published online: 27 August 2016}

\section{References}

1. Gahl WA, Boerkoel CF, Boehm M. The NIH Undiagnosed Diseases Program: bonding scientists and clinicians. Disse models \& mechanisms. 2012;5(1):3-5. doi:10.1242/dmm.009258

2. Gahl WA, Markello TC, Toro C, Fajardo KF, Sincan M, Gill F, et al. The National Institutes of Health Undiagnosed Diseases Program: insights into rare diseases. Genetics in medicine : official journal of the American College of Medical Genetics. 2012;14(1):51-9. doi:10.1038/gim.0b013e318232a005.

3. Gahl WA, Tifft CJ. The NIH Undiagnosed Diseases Program: lessons learned. Jama. 2011:305(18):1904-5. doi:10.1001/jama.2011.613.

4. $\mathrm{Ng} \mathrm{SB}$, Turner EH, Robertson PD, Flygare SD, Bigham AW, Lee C, et al. Targeted capture and massively parallel sequencing of 12 human exomes. Nature. 2009:461(7261):272-6. doi:10.1038/nature08250.

5. Ng SB, Bigham AW, Buckingham KJ, Hannibal MC, McMillin MJ, Gildersleeve $\mathrm{HI}$, et al. Exome sequencing identifies MLL2 mutations as a cause of Kabuki syndrome. Nat Genet. 2010:42(9):790-3. doi:10.1038/ng.646.

6. Chong JX, Buckingham KJ, Jhangiani SN, Boehm C, Sobreira N, Smith JD, et al. The Genetic Basis of Mendelian Phenotypes: Discoveries, Challenges, and Opportunities. Am J Hum Genet. 2015;97(2):199-215. doi:10.1016/j.ajhg. 2015.06.009

7. Yang Y, Muzny DM, Reid JG, Bainbridge MN, Willis A, Ward PA, et al. Clinical whole-exome sequencing for the diagnosis of mendelian disorders. $\mathrm{N}$ Engl J Med. 2013:369(16):1502-11. doi:10.1056/NEJMoa1306555.

8. Farwell KD, Shahmirzadi L, El-Khechen D, Powis Z, Chao EC, Tippin Davis B, et al. Enhanced utility of family-centered diagnostic exome sequencing with inheritance model-based analysis: results from 500 unselected families with undiagnosed genetic conditions. Genet Med. 2015;17(7):578-86. doi:10. 1038/gim.2014.154

9. Markello TC, Han T, Carlson-Donohoe H, Ahaghotu C, Harper U, Jones M et al. Recombination mapping using Boolean logic and high-density SNP genotyping for exome sequence filtering. Mol Genet Metab. 2012;105(3): 382-9. doi:10.1016/j.ymgme.2011.12.014.

10. MacArthur DG, Manolio TA, Dimmock DP, Rehm HL, Shendure J, Abecasis $G R$, et al. Guidelines for investigating causality of sequence variants in human disease. Nature. 2014;508(7497):469-76. doi:10.1038/nature13127.

11. Wright CF, Fitzgerald TW, Jones WD, Clayton S, McRae JF, van Kogelenberg $M$, et al. Genetic diagnosis of developmental disorders in the DDD study: a scalable analysis of genome-wide research data. Lancet. 2015;385(9975): 1305-14. doi:10.1016/S0140-6736(14)61705-0.

12. Robinson PN, Kohler S, Bauer S, Seelow D, Horn D, Mundlos S. The Human Phenotype Ontology: a tool for annotating and analyzing human hereditary disease. Am J Hum Genet. 2008;83(5):610-5. doi:10.1016/j.ajhg.2008.09.017.

13. Zhi D, Chen R. Statistical guidance for experimental design and data analysis of mutation detection in rare monogenic mendelian diseases by exome sequencing. PloS one. 2012;7(2):e31358. doi:10.1371/journal.pone.0031358.

14. Adams DR, Sincan M, Fuentes Fajardo K, Mullikin JC, Pierson TM, Toro C, et al. Analysis of DNA sequence variants detected by high-throughput sequencing. Human mutation. 2012;33(4):599-608. doi:10.1002/humu.22035.

15. Roach JC, Glusman G, Smit AF, Huff CD, Hubley R, Shannon PT, et al. Analysis of genetic inheritance in a family quartet by whole-genome sequencing. Science. 2010;328(5978):636-9. doi:10.1126/science.1186802.

16. Stenson PD, Mort M, Ball EV, Shaw K, Phillips A, Cooper DN. The Human Gene Mutation Database: building a comprehensive mutation repository for clinical and molecular genetics, diagnostic testing and personalized genomic medicine. Hum Genet. 2014;133(1):1-9. doi:10. 1007/s00439-013-1358-4.

17. Agrawal A, Hamvas A, Cole FS, Wambach JA, Wegner D, Coghill C, et al. An intronic $A B C A 3$ mutation that is responsible for respiratory disease. Pediatr Res. 2012;71(6):633-7. doi:10.1038/pr.2012.21.

18. Harland M, Mistry S, Bishop DT, Bishop JA. A deep intronic mutation in CDKN2A is associated with disease in a subset of melanoma pedigrees. Hum Mol Genet. 2001;10(23):2679-86.

19. Vache C, Besnard T, le Berre P, Garcia-Garcia G, Baux D, Larrieu L, et al. Usher syndrome type 2 caused by activation of an USH2A pseudoexon: implications for diagnosis and therapy. Hum Mutat. 2012;33(1):104-8. doi:10.1002/humu.21634.

20. Yu F, Lu J, Liu X, Gazave E, Chang D, Raj S, et al. Population genomic analysis of 962 whole genome sequences of humans reveals natural selection in non-coding regions. PloS One. 2015;10(3):e0121644. doi:10. 1371/journal.pone.0121644.

21. Mele C, Lemaire M, latropoulos P, Piras R, Bresin E, Bettoni S, et al. Characterization of a New DGKE Intronic Mutation in Genetically Unsolved Cases of Familial Atypical Hemolytic Uremic Syndrome. Clin J Am Soc Nephrol: CJASN. 2015;10(6):1011-9. doi:10.2215/CJN.08520814.

22. Guo Y, Long J, He J, Li Cl, Cai Q, Shu XO, et al. Exome sequencing generates high quality data in non-target regions. BMC Genomics. 2012;13:194. doi:10. 1186/1471-2164-13-194.

23. Kircher M, Witten DM, Jain P, O'Roak BJ, Cooper GM, Shendure J. A general framework for estimating the relative pathogenicity of human genetic variants. Nat Genet. 2014;46(3):310-5. doi:10.1038/ng.2892.

24. de Ligt J, Boone PM, Pfundt R, Vissers LE, Richmond T, Geoghegan J, et al. Detection of clinically relevant copy number variants with whole-exome sequencing. Hum Mutat. 2013:34(10):1439-48. doi:10.1002/humu.22387.

25. Fromer M, Moran JL, Chambert K, Banks E, Bergen SE, Ruderfer DM, et al. Discovery and statistical genotyping of copy-number variation from wholeexome sequencing depth. Am J Hum Genet. 2012;91(4):597-607. doi:10. 1016/j.ajhg.2012.08.005.

26. Plagnol V, Curtis J, Epstein M, Mok KY, Stebbings E, Grigoriadou S, et al. A robust model for read count data in exome sequencing experiments and implications for copy number variant calling. Bioinformatics. 2012;28(21): 2747-54. doi:10.1093/bioinformatics/bts526.

27. Le Scouarnec S, Gribble SM. Characterising chromosome rearrangements: recent technical advances in molecular cytogenetics. Heredity. 2012;108(1): 75-85. doi:10.1038/hdy.2011.100.

28. Challis D, Antunes L, Garrison E, Banks E, Evani US, Muzny D, et al. The distribution and mutagenesis of short coding INDELs from 1,128 whole exomes. BMC Genomics. 2015;16:143. doi:10.1186/s12864-015-1333-7.

29. Ye K, Schulz MH, Long Q, Apweiler R, Ning Z. Pindel: a pattern growth approach to detect break points of large deletions and medium sized insertions from paired-end short reads. Bioinformatics. 2009:25(21):2865-71. doi:10.1093/bioinformatics/btp394.

30. Abecasis GR, Altshuler D, Auton A, Brooks LD, Durbin RM, Gibbs RA, et al. A map of human genome variation from population-scale sequencing. Nature. 2010:467(7319):1061-73. doi:10.1038/nature09534.

31. Abecasis GR, Auton A, Brooks LD, DePristo MA, Durbin RM, Handsaker RE, et al. An integrated map of genetic variation from 1,092 human genomes. Nature. 2012;491(7422):56-65. doi:10.1038/nature11632.

32. Lelieveld SH, Spielmann M, Mundlos S, Veltman JA, Gilissen C. Comparison of Exome and Genome Sequencing Technologies for the Complete Capture 
of Protein-Coding Regions. Human Mutation. 2015;36(8):815-22. doi:10. 1002/humu.22813.

33. Meienberg J, Zerjavic K, Keller I, Okoniewski M, Patrignani A, Ludin K, et al. New insights into the performance of human whole-exome capture platforms. Nucleic Acids Res. 2015;43(11):e76. doi:10.1093/nar/gkv216.

34. Gudbjartsson DF, Helgason H, Gudjonsson SA, Zink F, Oddson A, Gylfason A, et al. Large-scale whole-genome sequencing of the Icelandic population. Nat Genet. 2015;47(5):435-44. doi:10.1038/ng.3247.

35. Green RC, Berg JS, Grody WW, Kalia SS, Korf BR, Martin CL, et al. ACMG recommendations for reporting of incidental findings in clinical exome and genome sequencing. Genet Med. 2013;15(7):565-74. doi:10.1038/gim.2013.73.

36. Park JY, Clark P, Londin E, Sponziello M, Kricka L, Fortina P. Clinical exome performance for reporting secondary genetic findings. Clin Chem. 2015; 61(1):213-20. doi:10.1373/clinchem.2014.231456.

37. Meynert AM, Ansari M, FitzPatrick DR, Taylor MS. Variant detection sensitivity and biases in whole genome and exome sequencing. BMC Bioinformatics. 2014;15:247. doi:10.1186/1471-2105-15-247.

38. Brand H, Pillalamarri V, Collins RL, Eggert S, O'Dushlaine C, Braaten EB, et al. Cryptic and complex chromosomal aberrations in early-onset neuropsychiatric disorders. Am J Hum Genet. 2014;95(4):454-61. doi:10. 1016/j.j.jhg.2014.09.005.

39. Gilissen C, Hehir-Kwa JY, Thung DT, van de Vorst M, van Bon BW, Willemsen $\mathrm{MH}$, et al. Genome sequencing identifies major causes of severe intellectual disability. Nature. 2014;511(7509):344-7. doi:10.1038/nature13394.

40. Usdin K. The biological effects of simple tandem repeats: lessons from the repeat expansion diseases. Genome research. 2008;18(7):1011-9. doi:10. 1101/gr.070409.107.

41. Zavodna M, Bagshaw A, Brauning R, Gemmell NJ. The accuracy, feasibility and challenges of sequencing short tandem repeats using next-generation sequencing platforms. PloS One. 2014;9(12), e113862. doi:10.1371/journal. pone. 0113862 .

42. English AC, Salerno WJ, Hampton OA, Gonzaga-Jauregui C, Ambreth S, Ritter DI, et al. Assessing structural variation in a personal genome-towards a human reference diploid genome. BMC Genomics. 2015;16:286. doi:10. 1186/s12864-015-1479-3.

43. Lv J, Liu H, Su J, Wu X, Liu H, Li B, et al. DiseaseMeth: a human disease methylation database. Nucleic Acids Res. 2012;40(Database issue):D1030-5. doi:10.1093/nar/gkr1169.

44. Robertson KD. DNA methylation and human disease. Nat Rev Genet. 2005; 6(8):597-610. doi:10.1038/nrg1655.

45. Solyom S, Kazazian Jr HH. Mobile elements in the human genome: implications for disease. Genome Med. 2012;4(2):12. doi:10.1186/gm311.

46. Bickmore WA. The spatial organization of the human genome. Annu Rev Genomics Hum Genet. 2013;14:67-84. doi:10.1146/annurev-genom$091212-153515$.

47. Dixon JR, Selvaraj S, Yue F, Kim A, Li Y, Shen Y, et al. Topological domains in mammalian genomes identified by analysis of chromatin interactions. Nature. 2012;485(7398):376-80. doi:10.1038/nature11082.

48. Lupianez DG, Kraft K, Heinrich V, Krawitz P, Brancati F, Klopocki E, et al. Disruptions of topological chromatin domains cause pathogenic rewiring of gene-enhancer interactions. Cell. 2015;161(5):1012-25. doi:10.1016/j.cell.2015.04.004.

49. Sobreira N, Schiettecatte F, Valle D, Hamosh A. GeneMatcher: A Matching Tool for Connecting Investigators with an Interest in the Same Gene. Human mutation. 2015. doi:10.1002/humu.22844.

50. Robinson PN, Kohler S, Oellrich A, Wang K, Mungall CJ, Lewis SE, et al. Improved exome prioritization of disease genes through cross-species phenotype comparison. Genome Res. 2014;24(2):340-8. doi:10.1101/gr.160325.113.

51. Buske OJ, Girdea M, Dumitriu S, Gallinger B, Hartley T, Trang H et al. PhenomeCentral: A Portal for Phenotypic and Genotypic Matchmaking of Patients with Rare Genetic Diseases. Human Mutat. 2015. doi:10.1002/ humu.22851.

52. Bone WP, Washington NL, Buske OJ, Adams DR, Davis J, Draper D, et al. Computational evaluation of exome sequence data using human and model organism phenotypes improves diagnostic efficiency. Genetics in medicine : official journal of the American College of Medical Genetics. 2015. doi:10.1038/gim.2015.137.

53. Kohler S, Doelken SC, Mungall CJ, Bauer S, Firth HV, Bailleul-Forestier I, et al. The Human Phenotype Ontology project: linking molecular biology and disease through phenotype data. Nucleic Acids Res. 2014;42(Database issue):D966-74. doi:10.1093/nar/gkt1026.
54. McKenna A, Hanna M, Banks E, Sivachenko A, Cibulskis K, Kernytsky A, et al. The Genome Analysis Toolkit: a MapReduce framework for analyzing nextgeneration DNA sequencing data. Genome Res. 2010;20(9):1297-303. doi:10. 1101/gr.107524.110

55. DePristo MA, Banks E, Poplin R, Garimella KV, Maguire JR, Hartl C, et al. A framework for variation discovery and genotyping using next-generation DNA sequencing data. Nat Genet. 2011;43(5):491-8. doi:10.1038/ng.806.

56. Van der Auwera GA, Carneiro MO, Hartl C, Poplin R, Del Angel G, LevyMoonshine A et al. From FastQ data to high confidence variant calls: the Genome Analysis Toolkit best practices pipeline. Current protocols in bioinformatics / editoral board, Andreas D Baxevanis [et al.]. 2013;11(1110): 110 1- 0 33. doi:10.1002/0471250953.bi1110s43.

57. Cingolani P, Platts A, le Wang L, Coon M, Nguyen T, Wang L, et al. A program for annotating and predicting the effects of single nucleotide polymorphisms, SnpEff: SNPs in the genome of Drosophila melanogaster strain w1118; iso-2; iso-3. Fly. 2012;6(2):80-92. doi:10.4161/fly.19695.

58. Farrell CM, O'Leary NA, Harte RA, Loveland JE, Wilming LG, Wallin C, et al. Current status and new features of the Consensus Coding Sequence database. Nucleic Acids Res. 2014;42(Database issue):D865-72. doi:10.1093/nar/gkt1059.

59. Pruitt KD, Harrow J, Harte RA, Wallin C, Diekhans M, Maglott DR, et al. The consensus coding sequence (CCDS) project: Identifying a common proteincoding gene set for the human and mouse genomes. Genome research. 2009;19(7):1316-23. doi:10.1101/gr.080531.108.

60. Harte RA, Farrell CM, Loveland JE, Suner MM, Wilming L, Aken B et al. Tracking and coordinating an international curation effort for the CCDS Project. Database : the journal of biological databases and curation. 2012; 2012:bas008. doi:10.1093/database/bas008.

61. Li H, Handsaker B, Wysoker A, Fennell T, Ruan J, Homer N, et al. The Sequence Alignment/Map format and SAMtools. Bioinformatics. 2009;25(16): 2078-9. doi:10.1093/bioinformatics/btp352.

62. Li H. A statistical framework for SNP calling, mutation discovery, association mapping and population genetical parameter estimation from sequencing data. Bioinformatics. 2011;27(21):2987-93. doi:10.1093/bioinformatics/btr509.

63. Karolchik D, Hinrichs AS, Furey TS, Roskin KM, Sugnet CW, Haussler D, et al. The UCSC Table Browser data retrieval tool. Nucleic Acids Res. 2004; 32(Database issue):D493-6. doi:10.1093/nar/gkh103.

64. Newcombe RG. Interval estimation for the difference between independent proportions: comparison of eleven methods. Stat Med. 1998;17(8):873-90.

\section{Submit your next manuscript to BioMed Central and we will help you at every step:}

- We accept pre-submission inquiries

- Our selector tool helps you to find the most relevant journal

- We provide round the clock customer support

- Convenient online submission

- Thorough peer review

- Inclusion in PubMed and all major indexing services

- Maximum visibility for your research

Submit your manuscript at www.biomedcentral.com/submit 